\title{
A comparative study of two pneumatic nebulizers for the treatment of Chronic Obstructive Pulmonary Disease (COPD); pre and post modification with polypropylene membrane
}

\author{
Lei Liu', Bin Liu ${ }^{2}$ \\ ${ }^{1}$ Department of Respiratory Medicine, the Fourth Affiliated Hospital of Harbin Medical University, Harbin 150001, China \\ ${ }^{2}$ The First Affiliated Hospital of Harbin Medical University, Harbin 150001, China
}

\begin{abstract}
Background and Objective: Pneumatic nebulizers (PN) are commonly used to treat COPD. We aimed to evaluate and compare two PNs with respect to effectiveness and delivery, and the effect of using a membrane filter to prevent drug wastage. Methods: COPD patients (240) were divided into an experimental group (EG); treated with a YZB/GEM1058-2009 medical atomizer, and a control group (CG); treated with a DNA100 medical atomizer. A polypropylene membrane was then applied to each nebulizer. Results: Wastage per inhalation was $79.56 \%$ in the EG, and $87 \%$ in the CG and reduced to $35.3 \%$ in the EG and $42.1 \%$ in the CG following application of a polypropylene membrane. The ratio of drug not atomised per inhalation was $10.32 \%$ in the EG, and $24.52 \%$ in the CG and altered to $30.2 \%$ and $37.3 \%$ with the polypropylene membrane. The total effective rate, cure rate and weekly efficiency were $96.7 \%, 73.3 \%$ and $93.3 \%$, respectively in the EG, and $93.3 \%, 60 \%$ and $83.3 \%$ respectively in the CG, and increased to $100 \%, 93.3 \%$ and $100 \%$, respectively in the $\mathrm{EG}$, and $100 \%, 83.3 \%$ and $93.3 \%$, respectively, in the CG with the membrane application. The number of days required to be effective was $4 \pm 2.4$ days in the EG, and $7 \pm 3.8$ in the CG and decreased to $3 \pm 1.4$ in the EG, and $5 \pm 3.1$ in the $C G$ with the membrane. Conclusions: There was a high percentage of drug wastage with both PNs. YZB/GEM1058-2009 was significantly more effective than DNA100 but a polypropylene microporous membrane significantly improved the effectiveness of both.
\end{abstract}

Keywords: Chronic Obstructive Pulmonary Disease; pneumatic nebulizer; drug waste; polypropylene microporous membrane

\section{Introduction}

COPD is a common and frequently occurring disease of the respiratory system. ${ }^{[1]} \mathrm{COPD}$ has risen to the fourth leading cause of mortality world-wide ${ }^{[2]}$. COPD represents an important public health challenge ${ }^{[3]}$.

Aerosol inhalation is a commonly used method for the clinical treatment of acute exacerbation of COPD. ${ }^{[4,5]}$ Pneumatic nebulizers are commonly used in clinics and are usually connected to the central oxygen supply system of the hospital; also called an oxygen nebulizer. ${ }^{[6]}$ Pneumatic atomizer models are diverse, however, there are no literature reports on the use of pneumatic nebulizers with respect to drug wastage. This study compared the effectiveness and drug wastage of two types of pneumatic nebulizers widely used in two large teaching hospitals.

Polypropylene microporous membrane is a non-toxic polymer material used as a membrane filter in a wide number

Copyright (C) 2018 Lei Liu et al.

doi: 10.18686/aem.v7i1.121

This is an open-access article distributed under the terms of the Creative Commons Attribution Unported License

(http://creativecommons.org/licenses/by-nc/4.0/), which permits unrestricted use, distribution, and reproduction in any medium, provided the original work is properly cited. 
of applications including medicine, chemicals, food, beverages and other fields ${ }^{[7]}$. We applied this polypropylene microporous membrane to the pneumatic nebulizer with the aim of reducing drug wastage, and further compared the two pneumatic nebulizer types following the application of this membrane.

\section{Methods}

This study was approved by the Harbin Medical University Medical Ethics Committee and having been informed of the purpose of the study, all patients agreed to participate. A total of 240 patients hospitalized with COPD exacerbations were selected from June 2016 to April 2017 in the Department of Respiration of the First and Fourth Affiliated Hospitals of Harbin Medical University. The experimental group (EG) and the control group (CG) both comprised 120 COPD patients from the Fourth Affiliated Hospital of Harbin Medical University. Both groups comprised 60 males and 60 females, and their age ranged from 55 to 65 years old. The average age was $61 \pm 5.4$ years in the EG group and $61 \pm 4.9$ years in the CG. The two groups of patients were comparable. Patients were excluded from the study if they did not cooperate with aerosol inhalation.

The 60 cases of EG were treated with the YZB/GEM1058-2009 medical atomizer (PARI GmbH, Germany), (Figure 1C). The 60 cases of CG were treated with the DNA100 medical atomizer (Chong Ren Medical Devices Co., Ltd Xiamen, China), (Figure 1C). The two nebulizers were connected to the central oxygen supply system of the hospital. The two groups of patients were treated with aerosol inhalation of budesonide suspension ( $2 \mathrm{mg}$ per treatment, AstraZeneca Pty Ltd, UK), a compound ipratropium bromide solution $(2.5 \mathrm{~mL}$ per treatment, Boehringer Ingelheim, Germany), and an ambroxol hydrochloride injection ( $15 \mathrm{mg}$ per treatment, Tianjin Pharmaceutical Research Institute Pharmaceutical Co., Ltd., China). The pressure outlet of the nebulizers was in contact with activated carbon particles (Wanjia Group Co., Ltd. China) that are able to adsorb the atomised drugs (Figure 1A and 1B). An electronic balance (ED224S-PCE, Sartorius, Germany) was used to weigh the drugs.

Nebulizer inhalation was conducted as follows: The nebulizer was placed next to the patient's bed and the air inlet of the nebulizer was connected to the central oxygen support system of the hospital. The suction nozzle was placed into the mouth of the patient, the pressure outlet of the nebulizer was connected to the activated carbon particles, and the central oxygen support system was opened at a flow rate of 5-6 L/min for $30 \mathrm{~min}$, (Figure 1A and 1B).

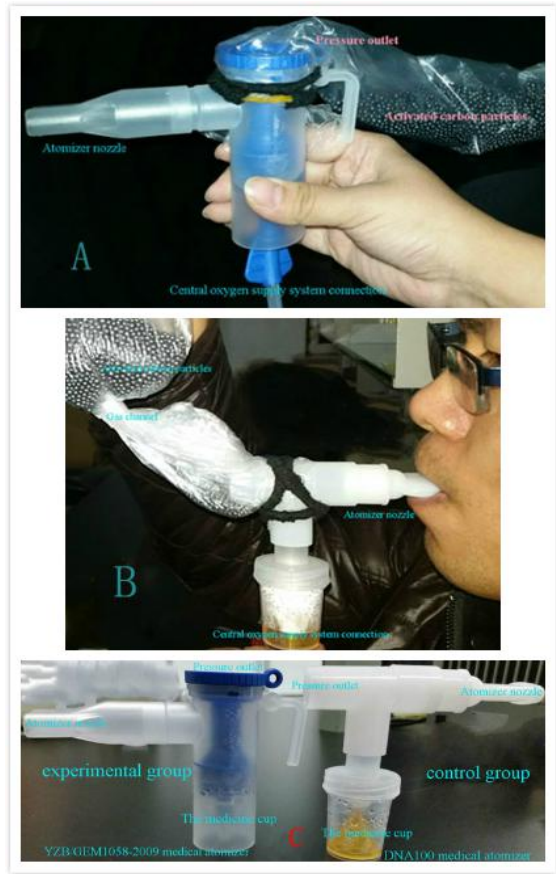

Figure 1, A; Working diagram of the nebulizer used in the experimental group.

Figure 1, B; Working diagram of the nebulizer in the control group.

Figure 1, C; A static display of the nebulizers in the experimental group and in the control group. 
The 60 cases of EG were treated with the YZB/GEM1058-2009 medical atomizer with a polypropylene microporous membrane modification. The 60 cases of CG were treated with the DNA100 medical atomizer with a polypropylene microporous membrane modification. The operation processes were the same as with application of a polypropylene microporous membrane (pore size, $1 \mu \mathrm{m}$, Hai-ning Yan-guan Long-shun Filtration Equipment Factory, Zhe-jiang Province, China) to the pneumatic nebulizer and the pressure outlet. (Figure 2D and 2E).

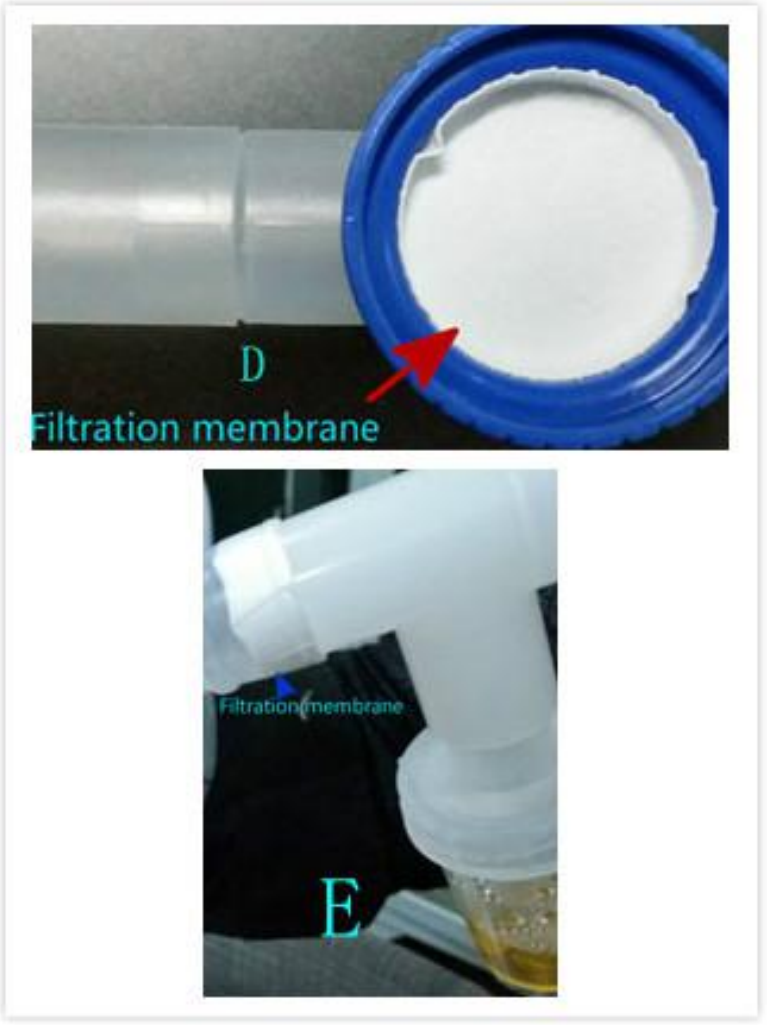

Figure 2, D; Working diagram of the nebulizer in the experimental group with polypropylene microporous membrane.

Figure 2, E; Working diagram of the nebulizer in the control group with polypropylene microporous membrane.

To calculate the average amount of drug wastage per inhalation we used the formula: the average amount of residual drug in the atomizer cup + the average amount of drug discharged into the air - the average amount of water vapour discharged by the human body with exhalation. Drug wastage ratio per inhalation = the average amount of drug wastage per inhalation / net weight of atomized medicine per inhalation $\times 100 \%$. The ratio of drug not atomised per inhalation $=$ the average amount of residual drug dose in atomizer cup per inhalation / net weight of atomized medicine per inhalation $\times 100 \%$. The average amount of money wasted per inhalation $=$ drug wastage ratio $\times$ the purchase fee per inhalation.

The criteria for efficiency were defined as recovery, i.e. if the patient's symptoms and lung wheeze completely disappeared, improvement, i.e., the patient's symptoms and lung wheeze improved significantly, and an invalid response, i.e., no improvement of symptoms and lung wheeze.

Total effective rate was calculated as [(number of cured cases + number of improved cases) / total number of cases] $\times 100 \%$. Cure rate $=($ number of cured cases $/$ total number of cases $) \times 100 \%$. Weekly efficiency was calculated as [(number of cured cases of aerosol inhalation weekly + number of improved cases of aerosol inhalation weekly) / total number of cases] $\times 100 \%$. The average time required to achieve an effective outcome (improved or cured) was calculated on a daily basis $(\mathrm{d})$.

Statistical methods: The measurement data were expressed as $\bar{x}_{ \pm \mathrm{s}}$, and the enumeration data were expressed as a percentage. SPSS17.0 software was used for statistical analysis $(\mathrm{P}<0.05$ was considered statistically significant). The measured results were in accord with the normal distribution, homogeneity of variance of measured values using Levene's Test. The $t$ test value was used if the variance was homogeneous, the Satterthwaite approximate $t$ test was used 
if the variance was not uniform, and the Chi square test value was used to compare the rate.

\section{Results}

The average net weight of the drugs per inhalation was $8.7984 \pm 0.0385$ grams.

The total cost of each inhalation was 7.18 USD and included 5.33 USD for the budesonide suspension, 0.91 USD for the compound ipratropium bromide solution and 0.94 USD for the ambroxol hydrochloride injection. The average amount of drug wastage per inhalation was $6.9998 \pm 0.004$ grams $(79.56 \%)$ for the EG, and $7.6546 \pm 0.0029$ grams $(87 \%)$ for the CG $(\mathrm{P}<0.001)$. The average ratio of drug not atomized per inhalation was $10.32 \%$ for the EG, and $24.52 \%$ for the CG. The total effective rate was $96.7 \%$ for the EG, and $93.3 \%$ for the $C G(P>0.05)$. The cure rate $(P<0.001)$ and weekly efficiency $(\mathrm{P}<0.05)$ were $73.3 \%$, and $93.3 \%$, respectively for the $\mathrm{EG}$, and $60 \%$, and $83.3 \%$, respectively for the $\mathrm{CG}$. The average number of days required to be effective was $4 \pm 2.4$ days for the $\mathrm{EG}$, and $7 \pm 3.8$ days for the CG $(\mathrm{P}<0.05$, Table 1).

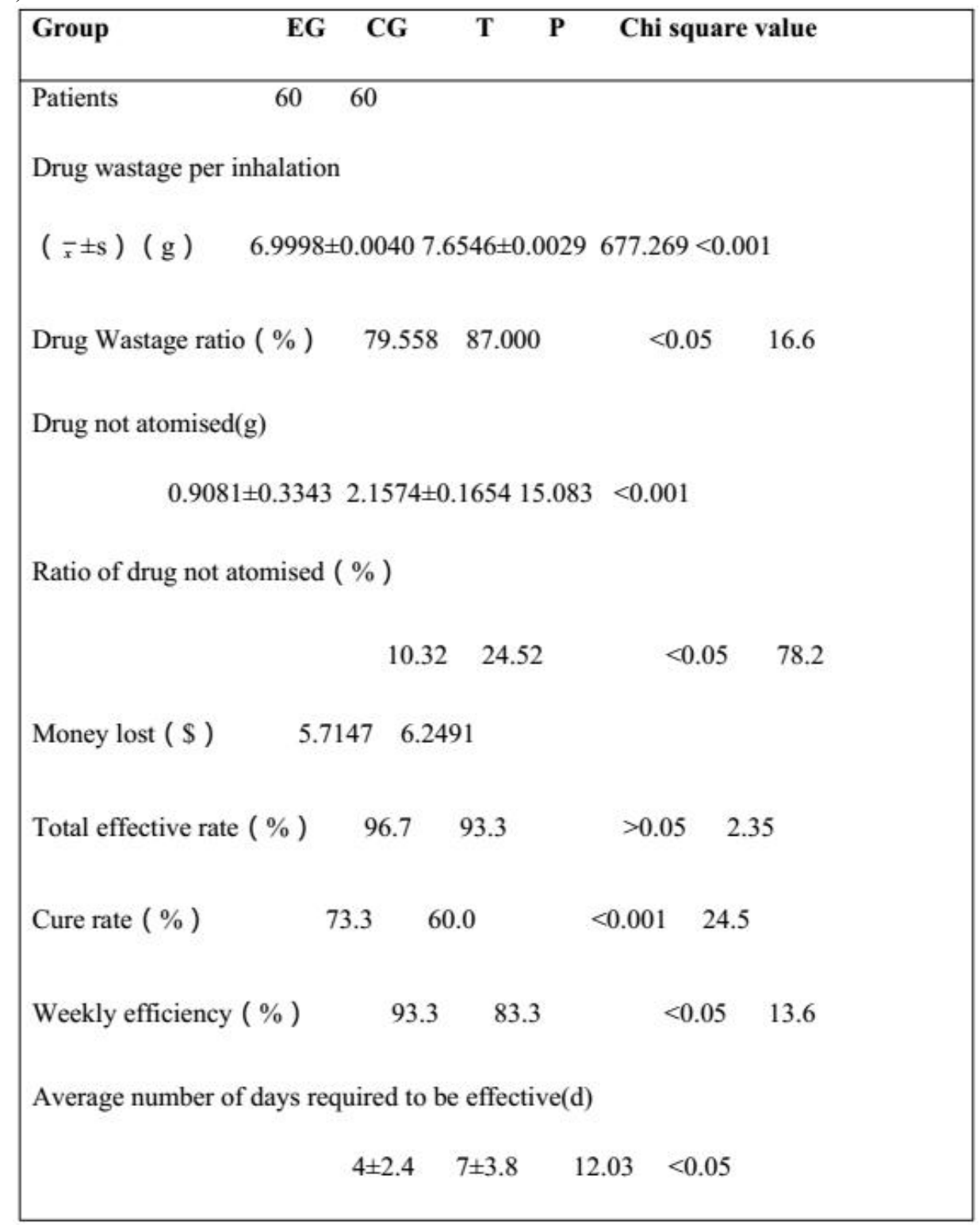

Table 1. Comparison of the experimental group(EG) and the control group(CG)

After application of the polypropylene microporous membrane the average amount of drug wastage per inhalation was reduced to $3.1032 \pm 0.025$ grams $(35.27 \%)$ in the $\mathrm{EG}$, and $3.7015 \pm 0.0339$ grams $(42.07 \%)$ in the $\mathrm{CG}(\mathrm{P}<0.001)$. The average amount of drug not atomised per inhalation process was $2.6571 \pm 0.1325$ grams $(30.2 \%)$ in the EG, and 
$3.2818 \pm 0.4234$ grams $(37.3 \%)$ in the $\mathrm{CG}(\mathrm{P}<0.001)$. The average amount of money wasted per inhalation was 0.32 USD in the EG and 0.39 USD in the CG. The total effective rate was $100 \%$ in the EG and $100 \%$ in the CG (P>0.05). The cure rate $(\mathrm{P}<0.05)$ and weekly efficiency $(\mathrm{P}<0.05)$ were $93.3 \%$ and $100 \%$, respectively in the $\mathrm{EG}$, and $83.3 \%$ and $93.3 \%$, respectively in the CG. The average number of days required to be effective was $3 \pm 1.4$ days in the EG and $5 \pm 3.1$ days in the $\mathrm{CG}$.

Thus, the use of the polypropylene membrane improved the total effective rate in the EG from $96.7 \%$ to $100 \%$ $(\mathrm{P}>0.05)$, the cure rate from $73.3 \%$ to $93.3 \%(\mathrm{P}<0.05)$, the weekly efficiency from $93.3 \%$ to $100 \%(\mathrm{P}<0.05)$, and the average number of days required to be effective from $4 \pm 2.4$ to $3 \pm 1.4(\mathrm{P}>0.05)$. In the $\mathrm{CG}$ total effective rate improved from $93.3 \%$ to $100 \%(\mathrm{P}<0.05)$, cure rate improved from $60 \%$ to $83.3 \%(\mathrm{P}<0.05)$, weekly efficiency from $83.3 \%$ to 93.3\% ( $<<0.05)$, and the average number of days required to be effective improved from $7 \pm 3.8$ to $5 \pm 3.1$ ( $\mathrm{P}<0.05)($ Table 3).

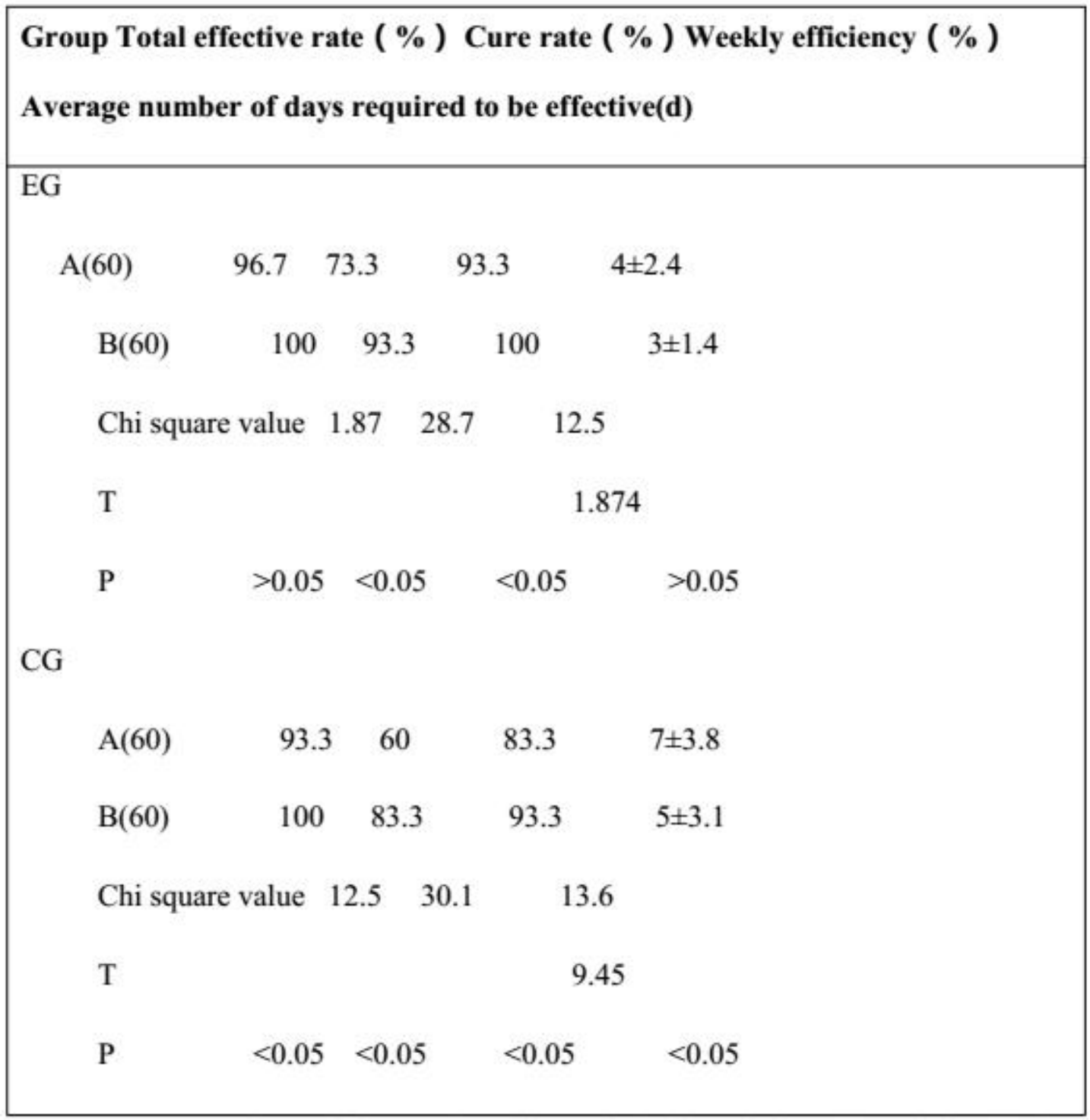

Table 3. Comparison of the curative effect of nebulizers before(A) and after(B) the application of the polypropylene membrane in the experimental group (EG) and control group (CG)

\section{Discussion}

Aerosol inhalation is undertaken via a specialised atomization device that sprays the medicinal solution or suspension into tiny droplets or particles that can be suspended as a gas and inhaled into the airways and alveoli to 
target lesions and treat disease. As it directly targets the lesions in the airway, the liver can be bypassed, unlike other medicinal applications. It also has the advantages of rapid onset, good curative effect, convenient use, less side effects, etc., and has thus become one of the most commonly used methods for the treatment of respiratory diseases. ${ }^{[4,5]}$

There are three types of medical nebulizers including the pneumatic nebulizer, the ultrasonic nebulizer, and the vibrating-mesh nebulizer. Oxygen delivery devices with a pneumatic nebulizer function are widely used in

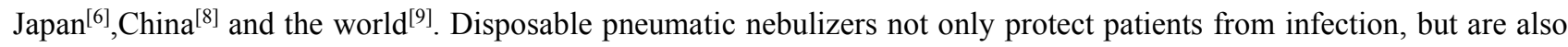
low cost and easy to use. Yin Xiao-Jing ${ }^{[8]}$ reported that treating the acute exacerbation of COPD with a pneumatic nebulizer not only improves blood oxygen saturation, but also alleviates the incidence of symptoms such as shortness of breath. The curative effect was superior to the medical ultrasonic atomizer.

Pneumatic nebulizers are currently the most commonly used inhalation method in clinical practice in China despite still having many shortcomings. The results of this study showed that the ability of the two different pneumatic nebulizers to atomize the drug was different. The aerodynamic atomizer used in the EG was more capable of atomizing the drug than the atomizer used in the CG where the amount of drug not atomized was significantly higher than in the EG. Thus, it would seem that the clinical selection of pneumatic nebulizer is important to avoid drug wastage.

Drug wastage in the EG was $79.56 \%$ and $87 \%$ in the CG. The cost of the drugs per inhalation was 7.18 USD, thus the amount wasted equated to a staggering 5.71 USD per inhalation in the EG and 6.24 USD in the CG. Similarly, our data on the effective rates, cure rates, weekly efficiency and number of days required to be effective also prompted us to look for better pneumatic nebulizers, and highlighted the need for more innovative designs. Disposable pneumatic nebulizers are currently widely used in clinical practice, and with atmospheric pollution levels, infection rates and airway disease rates as they are; there is a greater need for inhalation treatment and pneumatic nebulizers. Thus, better nebulizers, in particular pneumatic nebulizers, may have a significant economic benefit in China, and the rest of the world.

Polypropylene microporous membrane is a non-toxic polymer that is widely used in medicine, chemicals, food, beverages and other fields. We applied the polypropylene microporous membrane to the pneumatic nebulizer, resulting in a significant decrease in the amount of drugs, and money, wasted per inhalation in both groups. Similarly, all other factors tested were improved, i.e. the amount of drug not atomized, the total effective rate, the cure rate, the weekly efficiency and the average number of days required to be effective. The reduction in drug loss and amount of drug not atomised may have been because some of the droplets produced by the nebulizer were unable to pass through the membrane, and eventually attached to the wall of the pneumatic nebulizers. This minor modification resulted in a major improvement and would seem worth applying to the clinical setting.

\section{Conclusions}

The ability of the two different pneumatic nebulizers to atomise the drug differed; the YZB/GEM1058-2009 medical atomizer was significantly more capable of atomisation than the DNA100 medical atomizer. Thus, the clinical selection of pneumatic nebulizer is important to avoid drug wastage. With such a high rate of waste and such a large amount of money wasted in the inhalation process there is a need for better pneumatic nebulizers, and a call for more innovative designs.

We were able to significantly improve the delivery and effectiveness of two pneumatic nebulizers with the simple application of a polypropylene microporous membrane.

\section{References}

1. Rabe KF, Hurd S, Anzueto A, et al. Global initiative for chronic obstructive lung disease. Global strategy for the diagnosis, management, and prevention of chronic obstructive pulmonary disease: GOLD executive summary. Am. J. Respir. Crit. Care Med 2007; 176: 532-55.

2. (WHO) WHO. The top 10 causes of death [web page on the Internet. Geneva: WHO. http://www.who.int/gho/mortality_burden_disease/en/ [updated January 2017]. 
3. Global Initiative for Chronic Obstructive Lung Disease (GOLD). Global Strategy for the Diagnosis, Management and Prevention of COPD - 2016.

http://goldcopd.org/global-strategy-diagnosis-management-prevention-copd-2016/ Date last accessed: May 26, 2016. Date last updated: February 1, 2016.

4. Chen YQ, Li JP, Xiao J. Prophylactic effectiveness of budesonide inhalation in reducing postoperative throat complaints. Eur. Arch. Otorhinolaryngol 2014; 271: 1667-72.

5. Dolovich MB, Ahrens RC, Hess DR, et al. American College of Chest Physicians; American College of Asthma, Allergy, and Immunology. Device selection and outcomes of aerosol therapy: Evidence-based guidelines: American College of Chest Physicians/American College of Asthma, Allergy, and Immunology Chest 2005; 127 : 335-71.

6. Miyamoto K. An oxygen delivery device with a jet nebulizer function (Inspiron Nebulizer or Aquapak Nebulizer) can not provide high concentrations of oxygen to adult patients with respiratory failure. Nihon Kokyūki Gakkai Zasshi 2005; 43: 502.

7. Haocheng Yang, Junke Pi, Kun-Jian Liao, et al. Silica-Decorated polypropylene microfiltration membranes with a mussel-inspired intermediate layer for oil-in-water emulsion separation. ACS Appl. Mater. Interfaces 2014; 6(15): pp12566-12572.

8. XiaojingYin. Efficacy of oxygen inhalation in acute exacerbation of chronic obstructive pulmonary disease. World Latest Medicine Information 2016; 16: 265-6.

9. Dhand R. Aerosol delivery during mechanical ventilation from basic techniques to new devices. J. Aerosol. Med 2008; 21: 45-60. 